\title{
"Flipped Lab" Approach in Electronics Design to Enhance Student Learning Experience
}

\section{Dr. Nisha Kondrath, Villanova University}

Nisha Kondrath received her B.Tech degree in Electrical \& Electronics Engineering from Mahatma Gandhi University, Kerala, India in 2002 and M.S and Ph.D. degrees in Engineering from Wright State University, Dayton, OH, USA in 2005 and 2010, respectively. Dr. Kondrath is currently a Clare Boothe Luce Assistant Professor at the Department of Electrical and Computer Engineering, Villanova University, Villanova, PA, USA. Before joining Villanova University in August 2012, she was an Assistant Professor with the Department of Electrical and Computer Engineering, University of Minnesota Duluth, Duluth, MN, USA from August 2010-May 2012. Her research interests include power electronics, microgrids, PWM dcdc converters, PWM dc-ac inverters, power semiconductors, and high-frequency power magnetics. Ms. Kondrath is a member of IEEE, Tau Beta Pi, and ASEE.

\section{Dr. Mark A. Jupina, Villanova University}

Mark A. Jupina has been a professor in the Electrical and Computer Engineering Department at Villanova University since 1990. He has taught courses in electromagnetics, analog and digital electronics, and solid state materials, devices, and fabrication. Benjamin Franklin once said, "Tell me and I forget. Teach me and I remember. Involve me and I learn." This quote illustrates quite succinctly Mark Jupina's pedagogy in teaching courses to undergrads. Since the 1990's, Dr. Jupina has employed "active learning" techniques both inside and outside of the classroom using various modalities, including the flipped-lab approach during the last five years. More recently, Dr. Jupina has also incorporated Entrepreneurial Minded Learning (EML) exercises into his sophomore and junior-level courses. 


\title{
“FLIPPED LAB" APPROACH IN ELECTRONICS DESIGN TO ENHANCE STUDENT LEARNING EXPERIENCE
}

\author{
Department of Electrical \& Computer Engineering \\ Villanova University, Villanova, PA 19085
}

While no single pedagogy may work best all of the time when instructing students, we seek to create an exciting and dynamic learning environment to motivate students to become erudite self-learners in our rapidly changing world. Active and peer-to-peer learning strategies were employed both inside and outside of the lab environment in a two-course electronics sequence at the Department of Electrical and Computer Engineering at Villanova University. This flipped-lab approach was employed in an attempt to determine if this approach would (1) provide opportunities for faculty to challenge the students to perform more complex electronic circuit designs (2) foster more productive and student-centered peer-to-peer interactions, and (3) advance student abilities in the analysis, design, and testing of electronic circuits. This paper discusses the implementation of the pedagogy with examples of specific projects, faculty experiences and challenges, and student feedback with the new approach.

\section{Introduction}

Benjamin Franklin once said [1], "Tell me and I forget. Teach me and I remember. Involve me and I learn." As instructors, in addition to providing the technical knowledge, it is our responsibility to foster independent, critical, and creative thinking as well as encourage effective collaboration among students. To this end, we are presenting an updated pedagogy for the twocourse electronics sequence at the Department of Electrical and Computer Engineering at Villanova University to emphasize more self-discovery by a student before he or she engages in a structured, group-related lab activity.

Peer-to-peer learning strategies have been employed for a number of years at universities, such as Harvard and the University of Maryland [2], and self-learning techniques have even been tried at the local high school level [3]. Recently, the College of Engineering (CoE) has been taking initiatives in improving our students' learning experience at Villanova University. StudentCentered, Group-Related, Peer-to-Peer, or Active-Learning approaches were suggested as styles of instruction to consider. All of these approaches essentially involve less formal class lectures and more self-learning and discovery by a student while he or she works with other students in various projects. Conversations with other faculty and the director of Villanova Institute for Teaching and Learning (VITAL) at Villanova University also provided further understanding of successful techniques that engage students in an active learning environment. It was also emphasized that students prefer not to be lectured to and are more engaged (more motivated) in an active learning approach.

This approach was implemented in two electronics courses:

- Sophomore level "ECE 2550: Introduction to Electronics \& Applications" in the spring semesters of 2014-2017. 
- Junior level "ECE 3550: Analog Electronics" in the Fall semesters of 2014-2016. Lab modules using a Multisim circuit simulator and a myDAQ data acquisition unit from National Instruments were developed in an attempt to (1) provide opportunities for faculty to challenge the students to perform more complex electronic circuit designs, (2) foster more productive and student-centered peer-to-peer interactions, and (3) advance student abilities in the analysis, design, and testing of electronic circuits. These out-of-class activities provided more time for interactive active learning based problem sessions in class. Ultimately, this is expected to increase the efficiency of classroom activities and the effectiveness of the faculty's teaching in the limited formal class time. This paper discusses the implementation of the pedagogy with examples for specific projects, faculty experiences and challenges, and student feedback with the new approach.

\section{Pedagogical Approach}

Active learning strategies were employed both inside and outside of the lab environment in a two-course electronics sequence to develop more confident and motivated self-learners. This pedagogy emphasizes the advancement of an individual student's understanding on a certain topic outside of class before structured, group-related activities are performed in a lab setting. As a result, the students more effectively contribute to peer-to-peer learning, since each individual is well equipped to bring specific expertise to the group. Individual efforts combined with grouprelated activities provide a new dynamic whereby each activity type feeds off the other and produces motivated self-learners, who are also strong collaborative members of a team.

The course activities are divided into the following:

- Traditional lecture

The instructor delivered the technical content in the traditional lecture format.

- Active-learning problem sessions:

During this time, students are divided into groups and are asked to solve problems in class, typically at the end of the class session or periodically during the lecture. This activity encouraged teamwork and fostered critical thinking among students and improved their problem-solving skills. These sessions also gave the instructors an opportunity to reach out to individual students and get real-time feedback on students' understanding of the topic.

- Out-of-class DIY lab exercises:

Outside of the class meeting period, learning modules utilizing circuit simulations and portable circuit board-based lab measurements were assigned to encourage the student to learn at his or her own pace, wherever and whenever. Beyond individual learning sessions, open-lab periods monitored by a teaching assistant or staff were also conducted to allow the opportunity for students to tinker and explore on their own or within a group.

- In-class group lab exercises:

With significant individual prototyping and trouble-shooting completed outside of the class period, the instructors were able to focus on assessing the student's performance in real time through in-class group-related activities. Within the group, each student had different responsibilities for different aspects of the design. Each member has to design, test, and demonstrate his or her part before they can finish the project as a group. 
Consequently, a better determination of each student's level of absorption of the class materials was achieved. This also helped students to gain confidence in helping/mentoring their peers during the lab sessions and problem sessions.

\section{Implementation}

The student resources for these two courses include:

Textbook: "Microelectronics Circuits" by Sedra and Smith, 7th ed., Oxford University Press, 2014.

National instruments myDAQ data acquisition unit [5]

NI Multisim circuit simulation software

The topics covered, list of developed lab exercises, and sample lab assignments are presented in this section.

\section{ECE 2550: Intro to Electronics \& Applications:}

Implemented: Spring semesters 2014, 2015, 2016, 2017

Topics covered in this course include opamp circuits, diodes, diode circuits and applications, BJTs, BJT dc biasing circuits, and BJT amplifiers. Prior offerings of this course were scheduled such that one lecture (50 minutes) and one lab (2.5 hours) were conducted per week with the faculty being present at both sessions. Starting in the spring of 2013, ECE 2550 was scheduled for only one 2.5 hours session per week with the faculty being present to discuss lecture material and sample problems and to oversee the lab activity. Six new projects developed for ECE 2550, where each project involved both individual exercises and group-based exercises, are listed below:

Opamp amplifier circuit design (2 exercises)

Diode characteristics

BJT characteristics

BJT dc biasing circuits (Design and testing of 2 different biasing circuits)

$\mathrm{BJT}$ amplifier circuits (Design and testing of CE, CB)

The following two projects illustrate how the lab projects were structured.

\section{Sample Lab Exercises}

The purpose of the first project was for the students to use their understanding of basic op amp circuits to design a linear transfer function circuit which converts a DC input voltage over a certain range into a DC output voltage from 0 to $0.5,1$, or 1.7 volts. The linear transfer function circuit consisted of a non-inverting amplifier, a summing amplifier, and a voltage follower providing a DC reference voltage using a Zener diode. The objectives of this laboratory were to design, simulate, construct, and test the op amp circuit designs outside of the classroom and then assemble these sub-circuits in class to complete the final testing and verification of the entire design. Each design group was composed of either 2 or 3 students. Each student was responsible for designing, simulating, constructing, and testing a single op amp circuit in this design. For groups of two, each group member shared in the tasks for the voltage reference op amp circuit.

The second project objective was to design an electronic thermometer using three electronic components: a diode as a temperature sensor, a three op-amp circuit as a linear voltage converter, and a 555-timer IC configured as a voltage-controlled oscillator. As the sensed temperature 
changes, the frequency generated by the 555 timer varies by one octave. The specifications for each lab group was different such that the frequency range (somewhere in the one to ten Kilohertz range) was different for each group and half of the groups were required to generate low to high frequency tones as the temperature changes from cold to warm while the other half of the groups were to generate high to low frequency tones as the temperature changes from cold to warm. The diode was subjected to either a warm $\left(90^{\circ} \mathrm{F}\right)$ or cold $\left(40^{\circ} \mathrm{F}\right)$ bath of water. The diode junction voltage varies as a function of temperature, which was supplied as the input voltage to the three op-amp circuit. The three op-amp circuit creates a linear voltage function that converts the input voltage range to a different range of output voltages, which was fed into the input of the voltage-controlled oscillator circuit (555 timer). As the input voltage to the oscillator varies, the frequency of the tone generated by the 555-timer also varied. The students were able to listen to the varying frequency tones by placing a small speaker at the output of the 555-timer. Students performed this project in two-member groups. Both students in these two-member groups individually measured the current-voltage characteristics of a diode, so that each student could "calibrate" his or her own diode for temperature sensing.

These group projects involved interfacing the circuits designed by each individual. Each member was required to design and test the op-amp circuit or the timer circuit according to the given specifications outside the usual class period. Each individual was also required to explain the design specifics and the operational characteristics of his or her circuit to the other member in the group.

\section{ECE 3550: Analog Electronics:}

Implemented: Fall semesters 2014, 2015, 2016

Topics covered in this course include DC power supply, MOSFETs, MOSFET dc biasing circuits, MOSFET amplifiers, amplifier frequency response, and CMOS two-stage amplifiers. This four credit hour course was originally scheduled for three lectures (50 minutes each) and one lab (2.5 hours) sessions per week. Since Fall 2013, ECE 2550 has been scheduled for two 2.5 hours sessions per week. During each session, the faculty presents the lecture, conducts active learning problem sessions, and oversees lab activities. Six new lab exercises developed for this course are listed below:

DC power supply

MOSFET characteristics

MOSFET dc biasing circuits (Design and testing of 2 different biasing circuits)

MOSFET amplifier circuit (Design and testing of CS)

Frequency Characteristics of a CS amplifier

CMOS two-stage opamp

A sample project designed to enhance peer-to-peer learning and teamwork is described below.

\section{Sample Lab Exercise}

The objectives of this project were to build and test a two-stage CMOS based operational amplifier and then design a non-inverting or inverting amplifier with a specified gain. This experiment was performed in groups of two members. Both of them were to individually simulate and test the circuit. This helped the partners to gain an understanding of each stage of the CMOS op-amp. Hardware testing was divided into four steps: (1) Student 1 was to setup and 


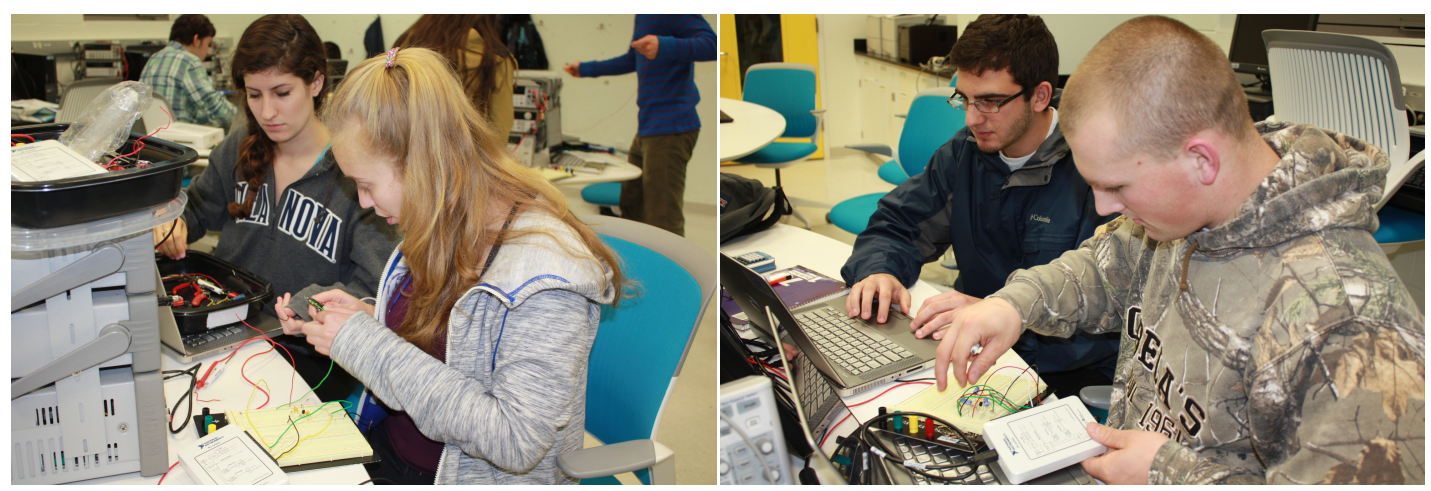

Figure 1. Students performing group activities in class using the myDAQ unit.

test the current-mirror biasing circuit. (2) Student 2 was to use the biasing circuit to build and test the differential amplifier, the first stage of the amplifier. (3) During the in-class session, each group continued to add the common-source amplifier, the second-stage, and test the two-stage CMOS operational amplifier. (4) Proceed to design, build, and test a non-inverting or inverting amplifier using the CMOS op-amp they built. During steps 2 and 3, the students understood the complexity of cascading different stages and how the individual stage gains affected the openloop gain of the op-amp. During the in-class demonstration, the students were asked questions regarding the operation of each stage to assess their understanding of the two-stage op-amp.

Lab projects instituted in this manner motivated the students to consider the big picture while designing the individual circuits, collaborating with their team members, and effectively contributing to peer-to-peer learning. Outside of the class activities including circuit simulations, prototyping, and testing helped advance the students' understanding in analysis, design, and troubleshooting of electronic circuits. Also, this allowed the faculty to dedicate more of the inclass time for interactive problem sessions.

\section{Faculty Experiences \& Challenges}

To improve the student motivation and performance, we need to increase the student investment in the course [4]. We believe that the active-learning approach implemented using the flipped-lab format shows promise in enabling the students to become better "self-starters," so as to better motivate themselves and their fellow classmates to take a more active role in their education. Oddly enough, that is also the most challenging part of this experience. The lab modules had to be well planned and organized with clear expectations of individual responsibilities. Getting the students to do some design work and circuit building outside of class on their own helped them to develop particular individual expertise in a specific area. Having the students share this particular knowledge with their peers in collaborative, group-based projects certainly built student confidence and enhanced students' skills in electronic circuit design. Group assignments designed in this manner also required considerable amount of teamwork, and it was important to ensure that each student was ready with their individual designs for the successful completion of the project.

Using the NI Multisim simulation software enabled the students to test and optimize their preliminary designs. The portable NI myDAQ unit helped the students to build and test the circuit prototypes in their dorm rooms at their leisure, rather than being restricted by 
the lab hour. However, experiments using the myDAQ unit outside of class sometimes got very time consuming because of the trouble-shooting issues and the glitches in the unit. During the spring of 2014, using the myDAQ module for the first time around, there was a big learning curve for both the students and the instructors. Now, after the third iteration, in both courses, lab exercises have been revised to address many common issues and problems of the module. For example, due to the output current ( $2 \mathrm{~mA}$ max) limitations of the function generator of the myDAQ, a voltage follower circuit is inserted when needed between the output of the generator and the test circuit to act as a buffer. Additional open-lab sessions monitored by the teaching assistant also provided additional help for the students.

Outside of the class activities including circuit simulations, prototyping, and testing helped advance the students' understanding in the analysis, design, and troubleshooting of electronic circuits. These out-of-class activities provided more time for interactive active-learning based interactive problem sessions in class. During the problem sessions, the instructor was able to engage the students, encourage peer-to-peer interaction, as well as assess and get immediate feedback on students' comprehension of the topics lectured during class. Ultimately, this increased the efficiency of classroom activities and the effectiveness of the instructor's teaching in the limited formal class time.

\section{Student Feedback}

At the end of Spring 2014 and midway through Spring 2017 semesters, surveys were conducted to obtain feedback from twenty-six students in 2014 and twenty-three students in 2017. These surveys contained questions concerning the flipped-lab pedagogy, the NI myDAQ measurement module, and the Multisim circuit simulation software. Nine opinion questions and four openended questions were included in the survey.

The nine opinion questions were the following:

1. I feel that the format of this course improved my overall learning compared to a traditional laboratory course.

2. I feel that the format of this course required a substantial amount more time compared to a traditional laboratory course.

3. The virtual measurements performed in the NI Multisim software were useful in providing an understanding of the circuit before performing actual measurements on the circuit with the NI myDAQ unit.

4. The NI myDAQ unit was a useful measurement tool.

5. Performing lab work outside of class with the myDAQ unit better prepared me for the group lab work in class.

6. Performing lab work outside of class with the myDAQ unit allowed me to learn at my own pace.

7. Performing lab work outside of class with the myDAQ unit has given me more confidence in performing lab measurements.

8. Performing lab work outside of the class with the myDAQ unit has enhanced my circuit prototyping and lab measurement skills.

9. I desire more technical courses where the lab work is performed individually outside of class. 

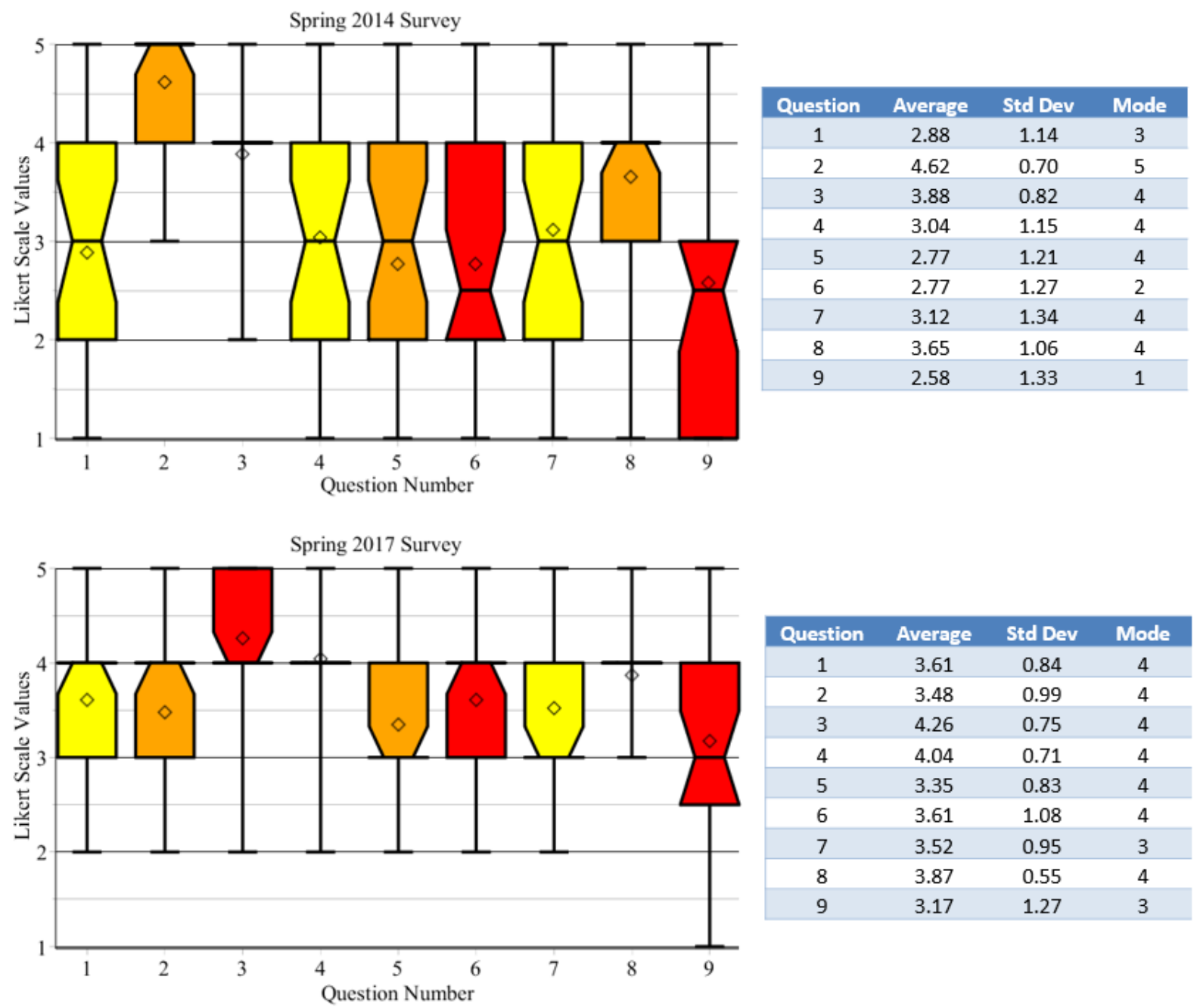

Figure 2. Summary of Statisical Results for the Likert Scale Responses

A summary of the statisical analysis on the nine opinion questions is shown in Figure 2 . The opinion questions were answered using a Likert scale: 1-strongly disagree, 2-disagree, 3-neutral, 4-agree, 5-strongly agree. The average (diamond symbol), median (notch on box), and first and third quartiles (thick longer lines at the ends of the box) are depicted in the Box and Whisker plots using the Likert scale data. For each question, the average, standard deviation, and the mode for the surveyed data is also given in tables next to each plot in Figure 2.

The four open ended questions in the student survey were the following:

10. What were some of the things that you liked about using the NI myDAQ and Multisim software?

11. What were some of the things that you disliked about using the NI myDAQ and Multisim software?

12. What were some of the things that you liked about the format of this course?

13. Is there anything you would change or improve about how this course was run? 
Even though a majority of students in 2014 responded negatively in the rating of question 6 (working at one's own pace), seven of the twenty-six students did comment in response to question 10 that they liked being able to perform both the measurements and simulations on their own, whenever and wherever they wanted to do these. The students did like the portability of the myDAQ unit and were initially surprised by the number of features available in such a small unit. A few students used the myDAQs to perform measurements in other courses. However, students' comments for question 11 reflected the many issues that arose during the semester with the myDAQs. Students felt that the NI myDAQ package was expensive $(\sim 200)$, the software was hard to install, the myDAQ was "buggy" and the measurement software crashed at times, and the myDAQ measurements were not always as reliable as the measurements performed with the lab equipment. In the responses to question 12, a few students commented that they were able to learn much more about the course topics by building and investigating the circuits by themselves. They also enjoyed learning the course material through a multi-faceted approach, where in-class lectures and the textbook readings supplemented the hands-on learning approach. However, students' comments for question 13 reflected the time commitment issue that was noted previously in the response of question 2. One student's comments summarized the general concensus of many students in the following way, "I think the course format has a lot of potential. Emphasizing personal problem solving and skill development is important and useful. I feel however that the course was a large time commitment, larger than I expected anyway. Working outside of class to learn has its advantages and I noticed these advantages while going through the course. However, I feel like I spent more time on this class than some of my core 3 and 4 credit courses that met three times a week, and that seems a bit imbalanced."

Based on the student feedback, the amount of material covered in the current offering of the introduction to electronics course in spring 2017 has been scaled back so that the time commitment by the students is more in line with a two credit course offering. More formal problem sessions are being administered, where students are asked to work on problems as a group and then present the solution to the class. This gives them an opportunity to develop their problem solving skills, build confidence, and promote peer-to-peer interactions.

When students were surveyed midway through the Spring 2017 semester, comparison of the boxand-whisker plots from 2014 and 2017 clearly show that adjustments made to the introduction to electronics course during the two-year interim were instrumental to enhancing the quality of the course. The increase in the scores for question 1 and questions 3 through 9 and the decrease in the score for question 2, clearly indicate that students in 2017 had a more positive experience in the course than the students in 2014 since their learning experience in electronics both inside and outside of classroom was improved. This improvement occurred through a restructuring of lab exercises because of an increased understanding of the myDAQ product and a better awareness of the student needs.

In regards to the student responses to the open-ended questions in 2017, the feedback was also more positive than in 2014. The surveyed responses to the tenth and twelfth questions showed that at least half of the students liked being able to do measurements outside of the lab with the myDAQ which allowed them to learn on their own and at their own pace. Beyond this advantage, two students commented that they liked using Multisim to perform a virtual measurement before performing the actual myDAQ measurement. Another student commented 
that the myDAQ allowed students to make measurements in different ways, which enhances the versatility in their measurement skills. Finally, several students enjoyed working through problems in groups in class after the lecture since it helped to reinforce their understanding of the material.

In the surveyed responses to the eleventh and thirteenth questions in 2017, a few students commented that the myDAQ was difficult to use at times due to connectivity issues or software issues and that there is an initial learning curve involved to using the product. The number of negative issues experienced with the myDAQ in 2017 is down from 2014 due a better understanding of the myDAQ unit by the instructors and better NI software, where fewer installation and reliability problems are now seen. Five students disliked the single two-and-ahalf hour class period per week and would prefer two shorter class periods per week. Other single comments noted concerns over the time involved in writing up the labs, performing labs outside of class, and using a myDAQ instead of lab bench equipment. However, overall in 2017 there are definitely fewer issues in regards to the myDAQs and the software as well as how the course is structured and offered. Consequently, we feel confident that future offerings of this course, introduction to electronics, and the follow-on course, analog electronics, are now well positioned to provide a positive learning experience for our students.

VI. Conclusions

A flipped-lab approach implemented in a two-course electronics sequence was discussed. Active learning strategies were employed both inside and outside of the classroom, where selfdiscovery exercises by a student were performed before the student engaged in a structured, group-related lab activity. Sample lab exercises in the sophomore and junior level electronics courses were presented. Analysis of the results of student surveys from Spring semesters of 2014 and 2017 were also presented. The first iteration of the flipped-lab approach in 2014 was met with relatively cold response from the students due to the additional time commitment involved using the myDAQ unit. However, as the course and lab exercises were refined, issues with the measurement unit were addressed, and the work load within the course was adjusted, many students saw the derived benefits of working with both the NI simulation software and measurement unit and preferred exploring and tinkering on their own as noted from 2017 survey. Also, the interactive problem sessions improved student engagement in the class, encouraged peer-to-peer interactions, amd nurtured critical thinking and were well-received by the students. Consequently, we feel confident that future offerings of this course, introduction to electronics, and the follow-on course, analog electronics, are now well positioned to provide a positive learning experience for our students. By providing more of such opportunities throughout the curriculum, we are confident that more students will develop the individual skills outside of the classroom to become better self-learners so that more productive peer-to-peer interactions within the classroom will lead to an enhanced learning environment.

\section{References}

[1] https://www.brainyquote.com/quotes/authors/b/benjamin_franklin.html, accessed online on Mar. 17, 2017.

[2] E. Hanford, Rethinking the way college students are taught, American Radio Works, September, 2011. http://americanradioworks.publicradio.org/features/tomorrows-college/lectures/rethinking-teaching.html

[3] K. A. Graham, Philadelphia sustainability workshop tries to reinvent the high school, Philadelphia Inquirer, 
Sunday, December 4, 2011.

http://articles.philly.com/2011-12-04/news/30474592_1_navy-yard-high-schools-school-day

[4] Stanford University Newsletter on Teaching, Active learning: Getting students to work and think in the classroom, vol. 5, no. 1, Fall 1993.

[5] http://www.ni.com/pdf/manuals/373060f.pdf, accessed online on Mar. 17, 2017.

[6] N. Kondrath and M. A. Jupina, "Combining individual lab work outside of class with group peer-to- peer learning in class to increase student ability in electronics design," in Proceedings of Mid-Atlantic ASEE Conference, Villanova, PA, Apr. 10-11, 2015. 